\title{
Controlling collapse in Bose-Einstein condensates by temporal modulation of the scattering length
}

\author{
Fatkhulla Kh. Abdullaev, ${ }^{1, *}$ Jean Guy Caputo, ${ }^{2, \dagger}$ Robert A. Kraenkel, ${ }^{1}$ and Boris A. Malomed ${ }^{1, \hbar}$ \\ ${ }^{1}$ Instituto de Fisica Teorica, UNESP, Rua Pamplona 145, 01405-900 São Paulo, Brazil \\ ${ }^{2}$ Laboratoire de Physique Théorique et Modelisation, Université de Cergy-Pontoise and CNRS, 33, boulevard de Pont, \\ 95011 Cergy-Pontoise, CEDEX, France
}

(Received 25 August 2002; published 21 January 2003)

\begin{abstract}
We consider, by means of the variational approximation (VA) and direct numerical simulations of the Gross-Pitaevskii (GP) equation, the dynamics of two-dimensional (2D) and 3D condensates with a scattering length containing constant and harmonically varying parts, which can be achieved with an ac magnetic field tuned to the Feshbach resonance. For a rapid time modulation, we develop an approach based on the direct averaging of the GP equation, without using the VA. In the 2D case, both VA and direct simulations, as well as the averaging method, reveal the existence of stable self-confined condensates without an external trap, in agreement with qualitatively similar results recently reported for spatial solitons in nonlinear optics. In the 3D case, the VA again predicts the existence of a stable self-confined condensate without a trap. In this case, direct simulations demonstrate that the stability is limited in time, eventually switching into collapse, even though the constant part of the scattering length is positive (but not too large). Thus a spatially uniform ac magnetic field, resonantly tuned to control the scattering length, may play the role of an effective trap confining the condensate, and sometimes causing its collapse.
\end{abstract}

DOI: 10.1103/PhysRevA.67.013605

PACS number(s): 03.75.Kk, 42.65. $-\mathrm{k}, 42.50 . \mathrm{Ar}$

\section{INTRODUCTION}

Collisions between atoms play a crucially important role in the dynamics of Bose-Einstein condensates (BECs). As is commonly known, the collisions are accounted for by the cubic term in the corresponding Gross-Pitaevskii (GP) equation that describes the BEC dynamics in the mean-field approximation. The coefficient in front of the cubic term, proportional to the collision scattering length, may be both positive and negative, which corresponds, respectively, to repulsive and attractive interactions between the atoms [1]. In the case of an attractive interaction, a soliton may be formed in an effectively one-dimensional (1D) condensate [2]; however, in $2 \mathrm{D}$ and $3 \mathrm{D}$ cases the attraction results in the collapse of the condensate (weak and strong collapse, respectively [3]) if the number of atoms exceeds a critical value [1].

Recently developed experimental techniques [4] make it possible to effectively control the sign of the scattering length using an external magnetic field because the interaction constant can be changed through the Feshbach resonance [5]. This technique makes it possible to quickly reverse (in time) the sign of the interaction from repulsion to attraction, which gives rise, via the onset of collapse, to an abrupt shrinking of the condensate, followed by a burst of emitted atoms and the formation of a stable residual condensate [4].

A natural generalization of this approach for controlling

\footnotetext{
*Permanent address: Physical-Technical Institute, Uzbek Academy of Sciences, 2-b Mavlyanov Strasse, 700084 Tashkent, Uzbekistan.

†Permanent address: Laboratoire de Mathematiques INSA de Rouen, Boîte Postal 8, 76131 Mont-Saint-Aignan cedex, France.

${ }^{\ddagger}$ Permanent address: Department of Interdisciplinary Studies, Faculty of Engineering, Tel Aviv University, Tel Aviv 69978, Israel.
}

the strength and sign of the interaction between atoms and, thus, the coefficient in front of the cubic term in the corresponding GP equation, is the application of a magnetic field resonantly coupled to the atoms and consisting, in the general case, of dc and ac components. The dynamical behavior of $2 \mathrm{D}$ and $3 \mathrm{D}$ condensates in this case is then an issue of straightforward physical interest, as it may be readily implemented in experiments. This is the subject of the present work.

It is worth noting that, in the 2D case, this issue is similar to a problem that was recently considered in nonlinear optics for $(2+1) D$ spatial solitons (i.e., self-confined cylindrical light beams) propagating across a nonlinear bulk medium with a layered structure, so that the size [6] and, possibly, the sign [7] of the Kerr (nonlinear) coefficient are subject to a periodic variation along the propagation distance (it plays the role of the evolutional variable, instead of time, in the description of optical spatial solitons). The same optical model makes also sense in the $(3+1) \mathrm{D}$ case, because it applies to the propagation of "light bullets" (3D spatiotemporal solitons [8]) through the layered medium [7]. We will demonstrate below that the results obtained for the BEC dynamics in the GP equation involving both dc and ac nonlinearity are indeed similar to findings reported in the framework of the above-mentioned optical model. To the best of our knowledge, a GP equation with a rapid time-periodic modulation of this type is proposed in this work for the first time. Previously, a quasi-1D model was considered in which the BEC stability was affected by a rapid temporal modulation applied to the trapping potential (rather than to the spatially uniform nonlinearity coefficient) [9] and the macroscopic quantum interference and resonances have been studied in Ref. [10]. Resonances in 2D and 3D BEC with periodically varying atomic scattering length have been considered in Refs. $[11,12]$. The main issue considered in this work is a possibility of self-localization of the condensate under the action 
of the ac field. In this connection, it is relevant to mention that the application of an external ac field can produce localization, in a very different sense, in linear quantum systems. In Ref. [13], it was demonstrated that a particle moving on a 1D lattice may fall into a localized state if it is driven by ac electric field, and in Ref. [14] it was predicted that dynamical localization, in the form of suppression of diffusion in a quantum system (periodically kicked rotor), whose classical counterpart demonstrates dynamical chaos, can be induced by external field. The latter effect was observed experimentally in an ultracold gas of two-level atoms, where the external field was a standing-wave created by a nearly resonant laser beam [15]. The paper is organized as follows. In Sec. II, we formulate the model to be considered in this work and the VA that will be employed to analyze the model. In Sec. III, variational and numerical results are presented for the 2D case (the analysis based on the VA also employs the Kapitsa averaging procedure). Both approaches demonstrate the existence of a stable self-sustained condensate, in a certain region of parameter space, so that the condensate can be effectively confined and maintained by means of a spatially uniform resonant ac magnetic field, without any trapping potential. In Sec. III, we also develop an alternative analytical approach, based on the application of the averaging procedure directly to the GP equation, without using the VA. Results produced by this approach confirm those obtained by means of the VA. In Sec. IV, we show that the results for the $3 \mathrm{D}$ case are essentially different from those in the 2D case. Here VA also predicts the possibility of a stable condensate, while direct simulations demonstrate that the stability is limited in time, finally giving way to collapse; a noteworthy fact is that, while the VA per se still provides reasonable results in the $3 \mathrm{D}$ case, the averaging procedure, if combined with the VA, may yield completely wrong predictions in this case. A nontrivial feature demonstrated by direct simulations in the $3 \mathrm{D}$ case is that the ac component of the nonlinearity may give rise to collapse even in the case when the dc (constant) component corresponds to repulsion. The paper is concluded in Sec. V.

\section{THE MODEL AND VARIATIONAL APPROXIMATION}

We take the mean-field GP equation for the single-particle wave function in its usual form,

$$
i \hbar \frac{\partial \psi}{\partial t}=-\frac{\hbar^{2}}{2 m} \Delta \psi+g|\psi|^{2} \psi,
$$

where $\Delta$ is the 2D or 3D Laplacian, $r$ is the corresponding radial variable, and $g=4 \pi \hbar^{2} a_{s} / m$, where $a_{s}, m$ are, respectively, the atomic scattering length and mass. As indicated above we will assume the scattering length to be time modulated so that the nonlinear coefficient in Eq. (1) takes the form $g=g_{0}+g_{1} \sin (\chi t)$, where $a_{0}$ and $a_{1}$ are the amplitudes of the dc and ac parts, and $\chi$ is the ac-modulation frequency.

Usually an external trapping potential is included to stabilize the condensate. We have omitted it because it does not play an essential role. This is also the case in some other situations, e.g., the formation of a stable Skyrmion in a two- component condensate [16]. In fact, we will demonstrate that the temporal modulation of the nonlinear coefficient, combining the dc and ac parts as in Eq. (2), may, in a certain sense, replace the trapping potential. Another caveat concerning the present model is that, if the frequency of the ac drive resonates with a transition between the ground state of the condensate and an excited quasiparticle state, the meanfield description based on the GP equation will not be adequate.

We now cast Eq. (1) in a normalized form by introducing a typical frequency $\Omega \sim 2 g n_{0} / \hbar$, where $n_{0}$ is the condensate density and rescale the time and space variables as $t^{\prime}=\Omega t$ $r^{\prime}=r \sqrt{2 m \Omega / \hbar}$. This leads to the following equation where primes have been omitted:

$$
i \frac{\partial \psi}{\partial t}=-\left(\frac{\partial^{2}}{\partial r^{2}}+\frac{D-1}{r} \frac{\partial}{\partial r}\right) \psi-\left[\lambda_{0}+\lambda_{1} \sin (\omega t)\right]|\psi|^{2} \psi,
$$

in which it is implied that $\psi$ depends only on $t$ and $r, D$ $=2$ or 3 is the spatial dimension, $\lambda_{0,1} \equiv-g_{0,1} /(\Omega \hbar), \omega$ $\equiv \chi / \Omega$.

Note that $\lambda_{0}>0$ and $\lambda_{0}<0$ in Eq. (2) correspond, respectively, to the self-focusing and self-defocusing nonlinearity. Rescaling the field $\psi$, we will set $\left|\lambda_{0}\right| \equiv 1$, so that $\lambda_{0}$ remains a sign-defining parameter.

The next step is to apply the VA to Eq. (2). This approximation was originally proposed [17] and developed in nonlinear optics first for 1D problems and later for multidimensional models (see a recent review [18]). A similar technique was elaborated for the description of the multidimensional BEC dynamics based on the GP equation [19].

To apply VA in the present case, we notice that the Lagrangian density generating Eq. (2) is

$$
\mathcal{L}(\psi)=\frac{i}{2}\left(\frac{\partial \psi}{\partial t} \psi^{*}-\frac{\partial \psi^{*}}{\partial t} \psi\right)-\left|\frac{\partial \psi}{\partial r}\right|^{2}+\frac{1}{2} \lambda(t)|\psi|^{4},
$$

where $\lambda(t) \equiv \lambda_{0}+\lambda_{1} \sin (\omega t)$, and the asterisk stands for the complex conjugation. The variational ansatz for the wave function of the condensate is chosen as the Gaussian [17],

$$
\psi_{g}(r, t)=A(t) \exp \left(-\frac{r^{2}}{2 a^{2}(t)}+\frac{1}{2} i b(t) r^{2}+i \delta(t)\right),
$$

where $A, a, b$, and $\delta$ are, respectively, the amplitude, width, chirp, and overall phase, which are assumed to be real functions of time. We did not include the degree of freedom related to the coordinate of the condensate's center, as the trapping potential, although not explicitly included into the model, is assumed to prevent the motion of the condensate as a whole.

Following the standard procedure [18], we insert the ansatz into the density (3) and calculate the effective Lagrangian,

$$
L_{\text {eff }}=C_{D} \int_{0}^{\infty} \mathcal{L}\left(\psi_{g}\right) r^{D-1} d r,
$$

where $C_{D}=2 \pi$ or $4 \pi$ in the $2 \mathrm{D}$ or $3 \mathrm{D}$ cases, respectively. Finally the evolution equations for the time-dependent pa- 
rameters of ansatz (4) are derived from $L_{\text {eff }}$ using the corresponding Euler-Lagrange equations. Subsequent analyses, as well as the results of direct numerical simulations, are presented separately for the 2D and 3D cases in Secs. III and IV.

\section{THE TWO-DIMENSIONAL CASE}

\section{A. Variational approximation}

In the 2D case, the calculation of the effective Lagrangian (5) yields

$$
L_{\mathrm{eff}}^{(2 \mathrm{D})}=\pi\left(-\frac{1}{2} a^{4} A^{2} \dot{b}-a^{2} A^{2} \dot{\delta}-A^{2}-a^{4} A^{2} b^{2}+\frac{1}{4} \lambda(t) a^{2} A^{4}\right),
$$

where the overdot stands for the time derivative. The EulerLagrange equations following from this Lagrangian yield the conservation of the number of atoms $N$ in the condensate,

$$
\pi A^{2} a^{2} \equiv N=\text { const, }
$$

an expression for the chirp and the width,

$$
\dot{a}=2 a b, \quad \dot{b}=\frac{2}{a^{4}}-2 b^{2}-\frac{\lambda(t) N}{2 \pi a^{4}},
$$

and a closed-form evolution equation for the width:

$$
\frac{d^{2} a}{d t^{2}}=\frac{2(2-\lambda(t) N / 2 \pi)}{a^{3}},
$$

which we rewrite as

$$
\begin{gathered}
\frac{d^{2} a}{d t^{2}}=\frac{-\Lambda+\epsilon \sin (\omega t)}{a^{3}}, \\
\Lambda \equiv 2\left(\lambda_{0} N /(2 \pi)-2\right), \epsilon \equiv-\lambda_{1} N / \pi .
\end{gathered}
$$

In the absence of an ac component, $\epsilon=0$, Eq. (9) conserves the energy $E_{2 \mathrm{D}}=\left(\dot{a}^{2}-\Lambda a^{-2}\right) / 2$. Obviously, $E_{2 \mathrm{D}}$ $\rightarrow-\infty$ as $a \rightarrow 0$, if $\Lambda>0$, and $E_{2 \mathrm{D}} \rightarrow+\infty$ as $a \rightarrow 0$, if $\Lambda$ $<0$. This means that, in the absence of the ac component, the 2D pulse is expected to collapse if $\Lambda>0$, and will spread out if $\Lambda<0$. The case $\Lambda=0$ corresponds to the critical number of particles in the condensate (the so-called "Townes soliton"). Note that a numerically exact value of the critical number is (in the present notation) $N=1.862$ [3], while the variational equation (10) yields $N=2$ (if $\lambda_{0}=+1$ ).

It is natural to specially consider the case when the ac component of the nonlinear coefficient oscillates at a high frequency. In this case, Eq. (9) can be treated analytically by means of the Kapitsa averaging method. To this end, we set $a(t)=\bar{a}+\delta a$, with $|\delta a| \ll|\bar{a}|$, where $\bar{a}$ varies on a slow time scale and $\delta a$ is a rapidly varying function with a zero mean value. After straightforward manipulations, we derive the following equations for the slow and rapid variables:

$$
\frac{d^{2}}{d t^{2}} \bar{a}=-\Lambda\left(\bar{a}^{-3}+6 \bar{a}^{-5}\left\langle\delta a^{2}\right\rangle\right)-3 \epsilon\langle\delta a \sin (\omega t)\rangle \bar{a}^{-4},
$$

$$
\frac{d^{2}}{d t^{2}} \delta a=3 \delta a \Lambda \bar{a}^{-4}+\epsilon \sin (\omega t) \bar{a}^{-3}
$$

where $\langle\cdots\rangle$ stands for averaging over the period $2 \pi / \omega$. A solution to Eq. (12) is

$$
\delta a(t)=-\frac{\epsilon \sin (\omega t)}{\bar{a}^{3}\left(\omega^{2}+3 \bar{a}^{-4} \Lambda\right)},
$$

the substitution of which into Eq. (11) yields the final evolution equation for the slow variable,

$$
\frac{d^{2}}{d t^{2}} \bar{a}=\bar{a}^{-3}\left[-\Lambda-\frac{3 \Lambda \epsilon^{2}}{\left(\omega^{2} \bar{a}^{4}+3 \Lambda\right)^{2}}+\frac{3}{2} \frac{\epsilon^{2}}{\omega^{2} \bar{a}^{4}+3 \Lambda}\right] .
$$

To examine whether collapse is enforced or inhibited by the ac component of the nonlinearity, one may consider Eq. (14) in the limit $\bar{a} \rightarrow 0$. In this limit, the equation reduces to

$$
\frac{d^{2}}{d t^{2}} \bar{a}=\left(-\Lambda+\frac{\epsilon^{2}}{6 \Lambda}\right) \bar{a}^{-3} .
$$

It immediately follows from Eq. (15) that, if the amplitude of the high-frequency ac component is large enough, $\epsilon^{2}$ $>6 \Lambda^{2}$, the behavior of the condensate (in the limit of small $\bar{a}$ ) is exactly opposite to that which would be expected in the presence of the dc component only: in the case $\Lambda>0$, bounce should occur rather than collapse, and vice versa in the case $\Lambda<0$.

On the other hand, in the limit of large $\bar{a}$, Eq. (14) takes the asymptotic form $d^{2} \bar{a} / d t^{2}=-\Lambda \bar{a}^{-3}$, which shows that the condensate remains self-confined in the case $\Lambda>0$, i.e., if the number of atoms exceeds the critical value. This consideration is relevant if $\bar{a}$ though being large remains smaller than the limit imposed by an external trapping potential, should it be added to the model. Thus, these asymptotic results guarantee that Eq. (14) gives rise to a stable behavior of the condensate, both the collapse and decay (spreading out) being ruled out if

$$
\epsilon>\sqrt{6} \Lambda>0 .
$$

In the experiments, for example, with ${ }^{7} \mathrm{Li}$ with the critical number $\sim 1500$ atoms if we have initially 1800 atoms (i.e., $N / 2 \pi=2.2)$ to stabilize the condensate, this means that the atomic scattering length for $\lambda_{0}=1$ should be harmonically modulated with the amplitude $\epsilon=0.98$. In fact, conditions (16) ensure that the right-hand side of Eq. (14) is positive for small $\bar{a}$ and negative for large $\bar{a}$. This implies that Eq. (14) 
must give rise to a stable fixed point (FP). Indeed, when conditions (16) hold, the right-hand side of Eq. (14) vanishes at exactly one FP,

$$
\omega^{2} \bar{a}^{4}=\frac{3 \epsilon^{2}}{4 \Lambda}+\sqrt{3\left(\frac{3 \epsilon^{4}}{16 \Lambda^{2}}-1\right)}-3 \Lambda,
$$

which can be easily checked to be stable through the calculation of an eigenfrequency of small oscillations around it.

Direct numerical simulations of Eq. (9) produce results (not shown here) that are in exact correspondence with those provided by the averaging method, i.e., a stable state with $a(t)$ performing small oscillations around point (17). The 3D situation shows a drastic difference because this correspondence breaks down, as shown in the following section.

For the sake of comparison with the results obtained by means of an alternative approach in Sec. III B, we also need an approximate form of Eq. (14) valid in the limit of small $\Lambda$ (i.e., when the number of atoms in the condensate is close to the critical value) and very large $\omega$ :

$$
\frac{d^{2}}{d t^{2}} \bar{a}=-\frac{\Lambda}{\bar{a}^{3}}+\frac{3}{2} \frac{\epsilon^{2}}{\omega^{2} \bar{a}^{7}} .
$$

To estimate the value of the amplitude of the highfrequency ac component necessary to stop the collapse, we note that a characteristic trap frequency is $\Omega \sim 100 \mathrm{~Hz}$. So, for a modulation frequency $\sim 3 \mathrm{kHz}$, which may be regarded as a typical "high modulation frequency," the dimensionless $\omega$ is $\sim 30$. If the initial dimensionless number of atoms is, for example, $N / 2 \pi=2.2$ so that according to Eq. (10), $\Lambda$ $=0.4$ (this corresponds to the ${ }^{7} \mathrm{Li}$ condensate with $\approx 1800$ atoms, the critical number being $\approx 1500$ ), and the parameters of modulation are $\lambda_{0}=1, \lambda_{1}=2.3, \epsilon=10$, then the stationary value of the condensate width found from Eq. (17) is $a_{s t}=0.8 l$, where $l=\sqrt{m \Omega / \hbar}$ is the healing length.

Thus our analytical approach, based on the VA and the subsequent use of the assumption that the number of atoms slightly exceeds the critical value, leads to an important prediction: in the 2D case, the ac component of the nonlinearity, acting jointly with the dc one corresponding to attraction, may give rise not to collapse, but rather to a stable solitonlike oscillatory condensate state that confines itself without the trapping potential. It is relevant to mention that a qualitatively similar result, viz., the existence of stable periodically oscillating spatial cylindrical solitons in a bulk nonlinear-optical medium consisting of alternating layers with opposite signs of the Kerr coefficient, was reported in Ref. [7], where this result was obtained in a completely analytical form on the basis of the VA, and was confirmed by direct numerical simulations.

\section{B. Averaging of the Gross-Pitaevskii equation and Hamiltonian}

In the case of a high-frequency modulation, there is a possibility to apply the averaging method directly to the $2 \mathrm{D}$ equation (2), without using the VA. Note that direct averaging was applied to the $2 \mathrm{D}$ nonlinear Schrödinger equation
(NLS) with a potential rapidly varying in space, rather than in time, in Ref. [20], where the main results were a renormalization of the parameters of the 2D NLS equation and a shift of the collapse threshold. As we will see below, a rapid temporal modulation of the nonlinear term in the GP equation leads to some effects, which do not reduce to a renormalization. Some nonlinear-dispersive and higher-order nonlinear terms will appear in the corresponding effective NLS equation [see Eq. (22) below]. These terms essentially affect the dynamics of the collapsing condensate.

Assuming that the ac frequency $\omega$ is large, we rewrite the 2D equation (2) in a more general form,

$$
i \partial \psi / \partial t+\Delta \psi+\lambda(\omega t)|\psi|^{2} \psi=0,
$$

where $\Delta$ is the 2D Laplacian. To derive an equation governing the slow variations of the field, we use the multiscale approach, writing the solution as an expansion in powers of $1 / \omega$ and introducing the slow temporal variables, $T_{k}$ $\equiv \omega^{-k} t, k=0,1,2, \ldots$, while the fast time is $\zeta \equiv \omega t$. Thus, the solution is sought for as

$$
\psi(r, t)=A\left(r, T_{k}\right)+\omega^{-1} u_{1}(\zeta, A)+\omega^{-2} u_{2}(\zeta, A)+\cdots,
$$

with $\left\langle u_{k}\right\rangle=0$, where $\langle\cdots\rangle$ stands for the average over the period of the rapid modulation, and we assume that $\lambda_{0}=$ +1 (i.e., the dc part of the nonlinear coefficient corresponds to attraction between the atoms).

Following a procedure developed, for a similar problem, in Ref. [21], we first find the first and second corrections,

$$
u_{1}=-i\left[\mu_{1}-\left\langle\mu_{1}\right\rangle\right]|A|^{2} A, \quad \mu_{1} \equiv \int_{0}^{\zeta}\left[\lambda(\tau)-\left\langle\lambda_{1}\right\rangle\right] d \tau,
$$

$$
\begin{aligned}
u_{2}= & {\left[\mu_{2}-\left\langle\mu_{2}\right\rangle\right]\left[2 i|A|^{2} A_{t}+i A^{2} A_{t}^{*}+\Delta\left(|A|^{2} A\right)\right] } \\
& -|A|^{4} A\left\{\frac{1}{2}\left[\left(\mu_{1}-\left\langle\mu_{1}\right\rangle\right)^{2}-2 M\right]+\langle\lambda\rangle\left(\mu_{2}-\left\langle\mu_{2}\right\rangle\right)\right\} .
\end{aligned}
$$

Here $\quad \mu_{2}=\int_{0}^{\zeta}\left(\mu_{1}-\left\langle\mu_{1}\right\rangle\right) d s, M=(1 / 2)\left(\left\langle\mu_{1}^{2}\right\rangle-\left\langle\mu_{1}\right\rangle^{2}\right)$ $=(1 / 2)\left(\left\langle\lambda^{2}\right\rangle-1\right)$ (recall we have set $\left.\left|\lambda_{0}\right|=1\right)$. Using these results, we obtain the following evolution equation for the slowly varying field $A\left(x, T_{0}\right)$, derived at the order $\omega^{-2}$ :

$$
\begin{aligned}
i \frac{\partial A}{\partial t}+ & \Delta A+|A|^{2} A+2 M\left(\frac{\epsilon}{\omega}\right)^{2}\left[|A|^{6} A-3|A|^{4} \Delta A\right. \\
& +2|A|^{2} \Delta\left(|A|^{2} A\right)+A^{2} \Delta\left(|A|^{2} A^{*}\right]=0,
\end{aligned}
$$

where $\epsilon$ is the same amplitude of the ac component as in Eq. (10). We stress that Eq. (22) is valid in both $2 \mathrm{D}$ and $3 \mathrm{D}$ cases. In either case, it can be represented in the quasiHamiltonian form

$$
\left[1+6 M\left(\frac{\epsilon}{\omega}\right)^{2}|A|^{4}\right] \frac{\partial A}{\partial t}=-i \frac{\delta H_{q}}{\delta A^{*}},
$$




$$
\begin{aligned}
H_{q}= & \int d V\left[|\nabla A|^{2}-2 M\left(\frac{\epsilon}{\omega}\right)^{2}|A|^{8}-\frac{1}{2}|A|^{4}\right. \\
& \left.+4 M\left(\frac{\epsilon}{\omega}\right)^{2}\left|\nabla\left(|A|^{2} A\right)\right|^{2}\right],
\end{aligned}
$$

where $d V$ is the infinitesimal volume in the 2D or 3D space. To cast this result in a canonical Hamiltonian representation, one needs to properly define the corresponding symplectic structure (Poisson's brackets), which is not our aim here. However, we notice that, as it immediately follows from Eq. (23) and the reality of the (quasi-)Hamiltonian (24), $H_{q}$ is an integral of motion, i.e., $d H_{q} / d t=0$.

For a further analysis of the 2D case, we apply a modulation theory developed in Ref. [22]. According to this theory, the solution is searched for in the form of a modulated Townes soliton. The (above-mentioned) Townes soliton is a solution to the 2D NLS equation in the form $\psi(r, t)$ $=e^{i t} R_{T}(r)$, where the function $R_{T}(r)$ satisfies the boundary value problem

$$
R_{T}^{\prime \prime}+r^{-1} R_{T}^{\prime}-R_{T}+R_{T}^{3}=0, \quad R_{T}^{\prime}(0)=0, \quad R_{T}(\infty)=0 .
$$

For this solution, the norm $N$ and the Hamiltonian $H$ take the well-known values,

$$
\begin{gathered}
N_{T} \equiv \int_{0}^{\infty} R_{T}^{2}(r) r d r=N_{c} \equiv 1.862, \\
H_{T}=\int_{0}^{\infty}\left[\left(R_{T}^{\prime}\right)^{2}-\frac{1}{2} R_{T}^{4}(r)\right] r d r=0 .
\end{gathered}
$$

The averaged variational equation (22) indicates an increase of the critical number of atoms for the collapse, as opposed to the classical value (26). Using relation (20), we find

$$
N_{\text {crit }}=\int_{0}^{\infty}|\psi|^{2} r d r=N_{T}+2 M\left(\frac{\epsilon}{\omega}\right)^{2} I_{6},
$$

where $I_{6}=11.178$ (see Appendix A). This increase in the critical number of atoms is similar to the well-known energy enhancement of dispersion-managed solitons in optical fibers with periodically modulated dispersion [23,24].

Another nontrivial perturbative effect is the appearance of a nonzero value of the phase chirp inside the stationary soliton. We define the mean value of the chirp as

$$
b=\frac{\int_{0}^{\infty} \operatorname{Im}\left(\frac{\partial \psi}{\partial r} \psi^{*}\right) r d r}{\int_{0}^{\infty} r^{2} d r|\psi|^{2}}
$$

Making use of expression (21) for the first correction, we find

$$
\begin{gathered}
b=-\frac{\epsilon}{\omega} B M\left(\mu_{1}-\left\langle\mu_{1}\right\rangle\right) \\
B \equiv 3 \frac{\int_{0}^{+\infty} r d r R^{2}\left(R^{\prime}\right)^{2}-(0.25) \int_{0}^{+\infty} d r R^{4}}{\int_{0}^{+\infty} r^{2} d r R^{2}}=0.596 .
\end{gathered}
$$

To develop a general analysis, we assume that the solution with the number of atoms close to the critical value may be approximated as a modulated Townes soliton, i.e.,

$$
\begin{gathered}
A(r, t) \approx[a(t)]^{-1} R_{T}[r / a(t)] e^{i S}, \quad S=\sigma(t)+\frac{\dot{a} r^{2}}{4 a}, \\
\dot{\sigma}=a^{-2}
\end{gathered}
$$

with some function $a(t)$ (where the overdot stands for $d / d t$ ). If the initial power is close to the critical value, i.e., when $\left|N-N_{c}\right| \ll N_{c}$ and the perturbation is conservative, i.e.,

$$
\operatorname{Im} \int d V\left[A^{*} F(A)\right]=0
$$

as in our case, a method worked out in Ref. [22] makes it possible to derive an evolution equation for the function $a(t)$, starting from approximation (27). The equation of the modulation theory for width is

$$
a^{3} a_{t t}=-\beta_{0}+\frac{\epsilon^{2}}{4 M_{0} \omega^{2}} f_{1}(t)
$$

where

$$
\beta_{0}=\beta(0)-\frac{\epsilon^{2} f_{1}(0)}{4 M_{0} \omega^{2}}, \quad \beta(0)=\frac{\left(N-N_{c}\right)}{M_{0}},
$$

and $M_{0} \equiv(1 / 4) \int_{0}^{\infty} r^{3} d r R_{T}^{2} \approx 0.55$. The auxiliary function is given by

$$
f_{1}(t)=2 a(t) \operatorname{Re}\left[\frac{1}{2 \pi} \int d x d y F\left(A_{T}\right) e^{-i S}\left\{R_{T}+\rho \nabla R_{T}(\rho)\right\}\right] .
$$

In the lowest-order approximation, the equation takes the form (for the harmonic modulation)

$$
\frac{d^{2} a}{d t^{2}}=-\frac{\Lambda_{1}}{a^{3}}+\frac{C \epsilon^{2}}{\omega^{2} a^{7}},
$$

where $\Lambda_{1}=\left(N-N_{c}\right) / M_{0}-C \epsilon^{2} /\left(\omega^{2} a_{0}^{4}\right)$ and $C$ is

$$
C \equiv \frac{3}{M_{0}} \int_{0}^{\infty} d \rho\left[2 \rho R_{T}^{4}\left(R_{T}^{\prime}\right)^{2}-\rho^{2} R_{T}^{3}\left(R_{T}^{\prime}\right)^{3}-\frac{1}{8} \rho R_{T}^{8}\right] \approx 39 .
$$

Values of integrals are given in Appendix A. Thus the averaged equation predicts the arrest of collapse by the rapid 
modulations of the nonlinear term in the 2D GP equation. The comparison of Eq. (30) with its counterpart (18), which was derived by means of averaging the VA-generated equation (9), shows that both approaches lead to the same behavior near the collapse threshold. The numerical coefficients in the second terms are different due to the different profiles of the Gaussian and Townes soliton. In this connection, it is relevant to mention a recent work [25], that has demonstrated that, generally, one may indeed expect good agreement between results for 2D solitons produced by VA and by the method based on the modulated Townes soliton.

Let us estimate the value of the fixed point for the numerical simulations performed in Ref. [6]. In this work the stable propagation of solitons has been observed for two-step modulation of the nonlinear coefficient in 2D NLSE. The modulation of the nonlinear coefficient was $\lambda=1+\epsilon$ if $T$ $>t>0$, and $\lambda=1-\epsilon$ for $2 T>t>T$. The parameters in the numerical simulations have been taken as $T=\epsilon$ $=0.1, N /(2 \pi)=11.726 /(2 \pi)$, with the critical number $N_{c}$ $=11.68 /(2 \pi)$. The map strength is $M=\epsilon^{2} T^{2} / 24$. For this values we have $a_{c}=0.49$, which agreed with the value $a_{c}$ $\approx 0.56$ following from the numerical experiment.

Instead of averaging Eq. (2), one can apply the averaging procedure, also based on representation (20) for the wave function, directly to the Hamiltonian of Eq. (2). As a result, the averaged Hamiltonian is found in the form

$$
\begin{aligned}
\bar{H}= & \int d x d y\left[|\nabla A|^{2}+2 M\left(\frac{\epsilon}{\omega}\right)^{2}\left|\nabla\left(|A|^{2} A\right)\right|^{2}-\frac{1}{2}|A|^{4}\right. \\
& \left.-6 M\left(\frac{\epsilon}{\omega}\right)^{2}|A|^{8}\right] .
\end{aligned}
$$

A possibility to stop the collapse, in the presence of a rapid periodic modulation of the atomic scattering length, can be explained on the basis of this Hamiltonian. To this end, following the pattern of the usual virial estimates [3], we note that, if a given field configuration has compressed itself to a spot with a size $\rho$, where the amplitude of the $A$ field is $\sim \aleph$, the conservation of the number of particles, $N$, [which may be applied to the $A$ field through the relation (20)] yields the relation

$$
\aleph^{2} \rho^{D} \sim N
$$

(recall $D$ is the space dimension). On the other hand, the same estimate for the strongest collapse-driving and collapse-stopping terms [the fourth and second terms, respectively, in expression (32)] $H_{-}$and $H_{+}$in the Hamiltonian yields

$$
H_{-} \sim-\left(\frac{\epsilon}{\omega}\right)^{2} \aleph^{8} \rho^{D}, \quad H_{+} \sim\left(\frac{\epsilon}{\omega}\right)^{2} \aleph^{6} \rho^{D-2} .
$$

Eliminating the amplitude from Eqs. (34) by means of relation (33), we conclude that, in the case of the catastrophic self-compression of the field in the $2 \mathrm{D}$ space, $\rho \rightarrow 0$, both terms $H_{\mp}$ take the same asymptotic form, $\rho^{-6}$, hence the collapse may be stopped, depending on details of the configuration. However, in the 3D case the collapse-driving term
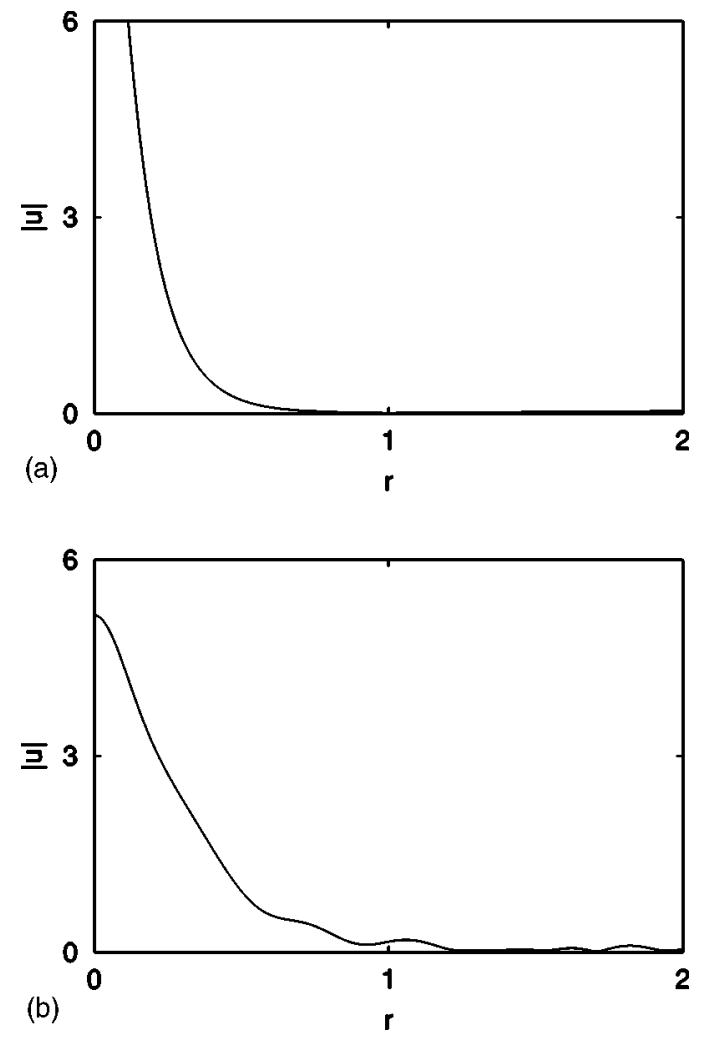

FIG. 1. A typical example of the formation of a self-confined condensate, revealed from direct simulations of Eq. (2) in the twodimensional case. Panel (a) shows pulse collapse in the absence of modulation for $t \approx 0.3$. Panel (b) shows the modulated pulse with the same initial condition for $t \approx 0.6$. The parameters are $\lambda_{0}=2.4$, $\lambda_{1}=0.85, \omega=100 \pi$, and $N=5$.

diverges as $\rho^{-9}$, while the collapse-stopping term has the asymptotic form $\sim \rho^{-8}$, for $\rho \rightarrow 0$, hence in this case the collapse, generally speaking, cannot be prevented.

Lastly, it is relevant to mention that, although the quasiHamiltonian (24) is not identical to the averaged Hamiltonian (32), the virial estimate applied to $H_{q}$ yields exactly the same result: the collapse can be stopped in the $2 \mathrm{D}$ but not in the $3 \mathrm{D}$ situation.

\section{Direct numerical results}

The existence of stable self-confined solitonlike oscillating condensate states, predicted above by means of analytical approximations for the case (16), when the dc part of the nonlinearity corresponds to attraction between the atoms, and the amplitude of the ac component is not too small, must be checked against direct simulations of the $2 \mathrm{D}$ equation (2). In fact, it was quite easy to confirm this prediction [in the case $\lambda_{0}=-1$, i.e., when the dc component of the nonlinearity corresponds to repulsion, the direct simulations always show a decay (spreading out) of the condensate, which also agrees with the above predictions].

A typical example of the formation of a self-confined condensate, supported by the combination of the self-focusing $\mathrm{dc}$ and sufficiently strong ac components of the nonlinearity in the absence of an external trap, is displayed in Fig. 1. On 
the left panel we show the pulse collapse at $t \approx 0.3$ in the absence of modulation. In the presence of modulation the pulse is stabilized for about 40 periods after which it decays. Note the presence of radiation as the pulse adjusts to the modulation.

\section{THE THREE-DIMENSIONAL CASE}

\section{A. The variational approximation and averaging}

The calculation of the effective Lagrangian (5) in the 3D case yields

$$
\begin{aligned}
L_{\mathrm{eff}}^{(3 \mathrm{D})}= & \frac{1}{2} \pi^{3 / 2} A^{2} a^{3}\left[-\frac{3}{2} \dot{b} a^{2}-2 \dot{\delta}+\frac{1}{2 \sqrt{2}} \lambda(t) A^{2}\right. \\
& \left.-\frac{3}{a^{2}}-3 b^{2} a^{2}\right],
\end{aligned}
$$

cf. Eq. (6). The Euler-Lagrange equations applied to this Lagrangian yield the mass conservation,

$$
\pi^{3 / 2} A^{2} a^{3} \equiv N=\text { const, }
$$

an expression for the chirp,

$$
\dot{a}=2 a b, \quad \dot{b}=\frac{2}{a^{4}}-2 b^{2}-\frac{\lambda(t) N}{2 \sqrt{2} \pi^{3 / 2} a^{5}},
$$

and the evolution equation for the width of the condensate,

$$
\frac{d^{2} a}{d t^{2}}=\frac{4}{a^{3}}-\frac{\lambda(t)}{\sqrt{2} \pi^{3 / 2}} \frac{N}{a^{4}} .
$$

Note the difference of Eq. (36) from its 2D counterpart (8).

As in the $2 \mathrm{D}$ case, we renormalize the amplitudes of the $\mathrm{dc}$ and ac components of the nonlinearity, $\Lambda$ $\equiv 2^{-1 / 2} \pi^{-3 / 2} \lambda_{0} N$ and $\epsilon \equiv-2^{-1 / 2} \pi^{-3 / 2} \lambda_{1} N$, and cast Eq. (36) in the normalized form,

$$
\frac{d^{2} a}{d t^{2}}=\frac{4}{a^{3}}+\frac{-\Lambda+\epsilon \sin (\omega t)}{a^{4}} .
$$

In the absence of the ac term, $\epsilon=0$, Eq. (37) conserves the energy

$$
E_{3 \mathrm{D}}=\frac{1}{2} \dot{a}^{2}+2 a^{-2}-\frac{1}{3} \Lambda a^{-3} .
$$

Obviously, $E_{3 \mathrm{D}} \rightarrow-\infty$ as $a \rightarrow 0$, if $\Lambda>0$, and $E_{3 \mathrm{D}} \rightarrow+\infty$ if $\Lambda<0$, hence one will have collapse or decay (spreading out) of the pulse, respectively, in these two cases.

Prior to applying the averaging procedure (as it was done above in the 2D case), we solved Eq. (37) numerically, without averaging, to show that (within the framework of VA) there is a region in parameter space where the condensate, which would decay under the action of the repulsive dc nonlinearity $(\Lambda<0)$, may be stabilized by the ac component of the nonlinearity, provided that its amplitude is sufficiently large. To this end, we employed the variable-step ordinary

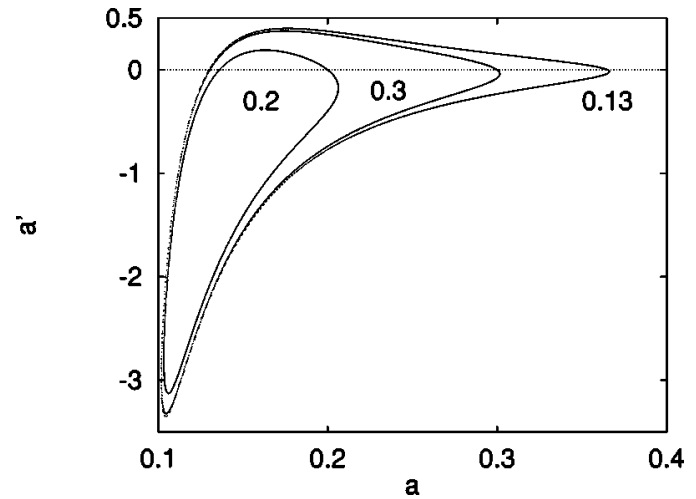

FIG. 2. The Poincaré section in the plane $(a, \dot{a})$ for $\Lambda=-1$, $\epsilon=100, \omega=10^{4} \dot{\pi}$, generated by the numerical solution of the variational equation (37) with different initial conditions (see the text).

differential equation (ODE) solver DOPRI5 [26], which is a combination of the Runge-Kutta algorithm of the fourth and fifth orders, so that the instantaneous truncation error can be controlled.

In Fig. 2 we show the dynamical behavior of solutions to Eq. (37), in terms of the Poincaré section in the plane $(a, \dot{a})$, obtained for $\Lambda=-1, \epsilon=100, \omega=10^{4} \pi$, and initial conditions $a(t=0)=0.3,0.2$, or 0.13 and $\dot{a}(t=0)=0$. As it is obvious from Fig. 2, in all these cases the solution remains bounded and the condensate does not collapse or decay, its width performing quasiperiodic oscillations.

In fact, the corresponding stability region in the parameter plane $(\omega / \pi, \epsilon)$ is small, see Fig. 3. It is also seen from Fig. 3 that the frequency and amplitude of the ac component need to be large to yield this stability. Notice that, for frequencies larger than $10^{6} \pi$, the width of the condensate $a(t)$ assumes very small values in the course of the evolution (as predicted by the VA) so that collapse may occur in practice for the solution of the full equation (2).

The stability is predicted by the VA only for $\Lambda<0$, i.e., for a repulsive dc component of the nonlinearity. In the opposite case, the VA predicts solely collapse.

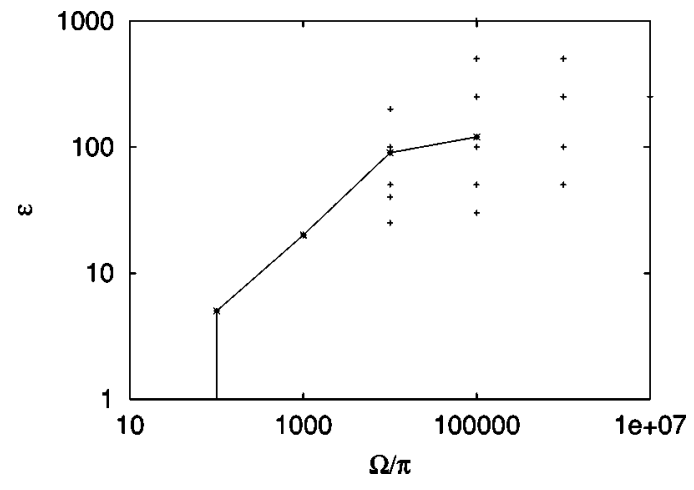

FIG. 3. The region in the $(\epsilon, \omega / \pi)$ parameter plane where the numerical solution of Eq. (37) with $\Lambda=-1$ predicts stable quasiperiodic solutions in the $3 \mathrm{D}$ case. Crosses mark points where stable solutions were actually obtained. Stars correspond to the minimum values of the ac-component's amplitude $\epsilon$ eventually leading to collapse of the solution of the full partial differential equation (2) with $\Lambda=-1$. 
As $\omega$ is large enough in the stability region shown in Fig. 3 , it seems natural to apply Kapitsa's averaging method to this case too. Doing it the same way as was described in detail in the preceding section for the $2 \mathrm{D}$ case, we find the rapidly oscillating correction $\delta a(t)$, cf. Eq. (13),

$$
\delta a=-\frac{\epsilon \sin (\omega t) \bar{a}}{\omega^{2} \bar{a}^{5}-12 \bar{a}+4 \Lambda},
$$

and then arrive at the evolution equation for the slow variable $\bar{a}(t)[$ cf. Eq. (14)]:

$$
\begin{aligned}
\frac{d^{2} \bar{a}}{d t^{2}}= & \bar{a}^{-4}\left[4 \bar{a}-\Lambda+\frac{2 \epsilon^{2}}{\omega^{2} \bar{a}^{5}-12 \bar{a}+4 \Lambda}\right. \\
& \left.+\epsilon^{2} \frac{6 \bar{a}-5 \Lambda}{\left(\omega^{2} \bar{a}^{5}-12 \bar{a}+4 \Lambda\right)^{2}}\right] .
\end{aligned}
$$

In the limit $\bar{a} \rightarrow 0$, Eq. (39) takes the form

$$
\frac{d^{2} \bar{a}}{d t^{2}}=\left(-\Lambda+\frac{3 \epsilon^{2}}{16 \Lambda}\right) \bar{a}^{-4},
$$

cf. Eq. (15). Equation (40) predicts one property of the 3D model correctly, viz., in the case $\Lambda<0$ and with a sufficiently large amplitude of the ac component $[\epsilon$ $>(4 / \sqrt{3})|\Lambda|$, as it follows from Eq. (40], collapse takes place instead of spreading out. However, other results following from the averaged equation (39) are wrong, as compared to those following from the direct simulations of the full variational equation (37), which were displayed abovesee Figs. 2 and 3. In particular, a detailed analysis of the right-hand side of Eq. (39) shows that it does not predict a stable FP for $\Lambda<0$, and does predict it for $\Lambda>0$, exactly opposite to what was revealed by the direct simulations. This failure of the averaging approach (in stark contrast with the $2 \mathrm{D}$ case) may be explained by the existence of singular points in Eqs. (38) and (39) (for both $\Lambda>0$ and $\Lambda<0$ ), at which the denominator $\omega^{2} \bar{a}^{5}-12 \bar{a}+4 \Lambda$ vanishes. Note that, in the 2D case with $\Lambda>0$, for which the stable state was found in the preceding section [see Eq. (16)], the corresponding Eq. (14) did not have singularities.

\section{B. Direct simulations of the Gross-Pitaevskii equation in the three-dimensional case}

Verification of the above results given by the VA against direct simulations of the 3D version of the radial equation (2) is necessary. The partial differential equation simulations were carried out by means of the method of lines implemented with the DOPRI5 ODE solver and space discretization involving high-order finite differences, see the details in Appendix $\mathrm{B}$. The relative error in the conservation of the number of atoms was limited by $10^{-8}$. In the absence of the ac modulation, the energy was conserved with a relative error limited by $10^{-8}$.

Quite naturally, in the case $\epsilon=0$ (no ac component) and $\Lambda<0$, the simulations show straightforward decay of the

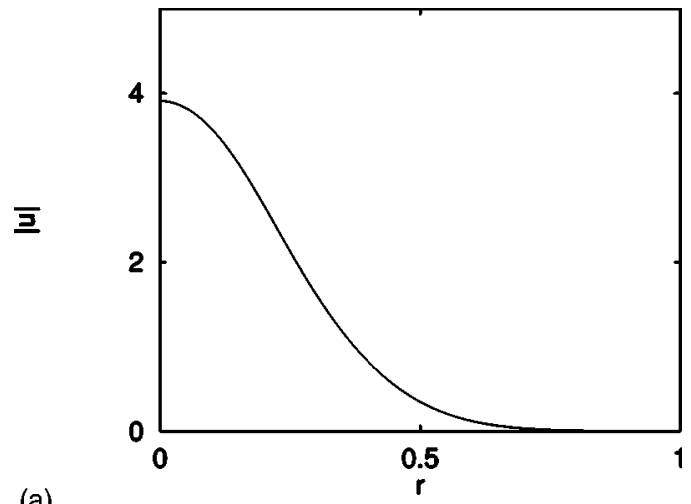

(a)

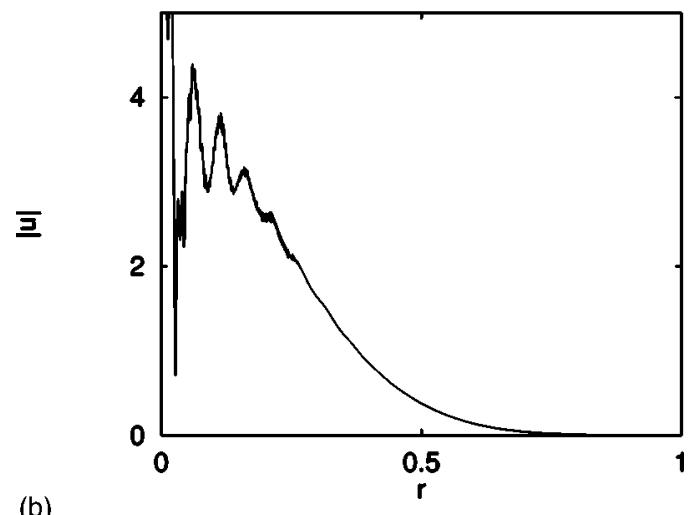

(b)

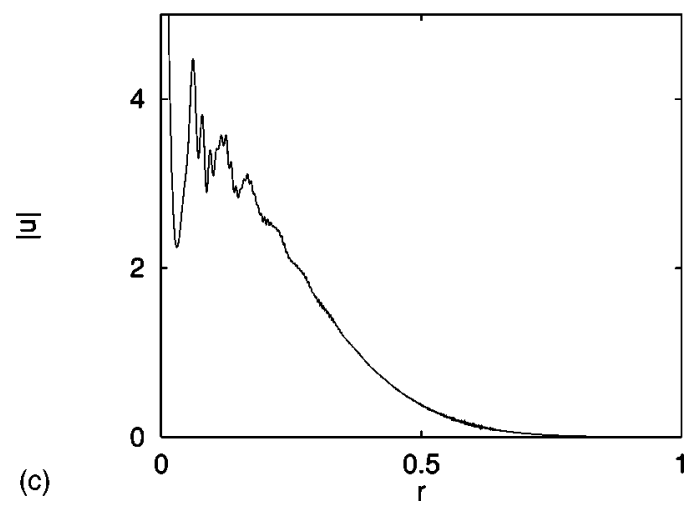

FIG. 4. Time evolution of the condensate's shape $|u|^{2}(r)$ in the presence of the strong and fast ac modulation $\left(\omega=10^{4} \dot{\pi}, \epsilon=90\right)$. The profiles of $u^{2}(r)$ are shown at times $t=0.007$ (a), 0.01 (b), and 0.015 (c)

condensate (not displayed here, as the picture is rather trivial). If an ac component of sufficiently large amplitude is added, stabilization of the condensate takes place temporarily, roughly the same way as is predicted by the solution of the variational equation (37). However, the stabilization is not permanent: the condensate begins to develop smallamplitude short-scale modulations around its center, and after about 50 periods of the ac modulation, it collapses.

An example of this behavior is displayed in Fig. 4, for which $N=1, \Lambda=-1$, and $\omega=10^{4} \pi$. Figure 4 shows radial profiles of the density $|u(r)|^{2}$ at different instants of time.

Results presented in Fig. 4 turn out to be quite typical for the $3 \mathrm{D}$ case with $\Lambda<0$. The eventual collapse that takes 
place in this case is a nontrivial feature, as it occurs despite the fact that the dc part of the nonlinearity drives the condensate towards spreading out. Therefore, a basic characteristic of the system is a dependence of the minimum ac amplitude $\epsilon$, which gives rise to the collapse at fixed $\Lambda=-1$, versus the ac frequency $\omega$. Several points marked by stars show this dependence in Fig. 3. It is quite natural that the minimum value of $\epsilon$ necessary for the collapse grows with $\omega$. On the other hand, for $\omega$ not too large, the minimum ac amplitude necessary for the onset of collapse becomes small, as even a small $\epsilon$ is sufficient to push the condensate into collapse during the relatively long half-period when the sign of the net nonlinearity coefficient $\lambda(t)$ is positive, see Eq. (19).

In the case of $\Lambda>0$ we have never been able to prevent the collapse of the pulse. This is in agreement with the analysis developed in the preceding section on the basis of the Hamiltonian of the averaged version of the GP equation, which showed that the collapse cannot be stopped in the 3D case, provided that the amplitude of the ac component is large enough. Besides that, this eventual result is also in accordance with the findings of direct simulations of the propagation of localized 3D pulses in the above-mentioned model of the nonlinear-optical medium consisting of alternating layers with opposite signs of the Kerr coefficient: contrary to the stable 2D spatial solitons [7], the 3D spatiotemporal "light bullets" can never be stable in this model [27].

\section{CONCLUSION}

In this work, we have studied the dynamics of $2 \mathrm{D}$ and $3 \mathrm{D}$ Bose-Einstein condensates in the case when the scattering length in the Gross-Pitaevskii (GP) equation contains constant (dc) and time-variable (ac) parts. This may be achieved in the experiment by means of a resonantly tuned ac magnetic field. Using the variational approximation (VA), simulating the GP equation directly, and applying the averaging procedure to the GP equation without the use of the VA, we have demonstrated that, in the $2 \mathrm{D}$ case, the ac component of the nonlinearity makes it possible to maintain the condensate in a stable self-confined state without external traps, which qualitatively agrees with recent results reported for spatial solitons in nonlinear optics. In the 3D case, VA also predicts a stable self-confined state of the condensate without a trap, provided that the constant part of the nonlinearity corresponds to repulsion between atoms. Direct simulations reveal that, in this case, the stability of the self-confined condensate is limited in time. Eventually, collapse takes place, despite the fact that the dc component of the nonlinearity is repulsive. Thus, we conclude that the spatially uniform ac magnetic field, resonantly tuned to affect the scattering length, may readily play the role of an effective trap that confines the condensate, and sometimes enforces its collapse. These predictions can be verified in experiments.

\section{ACKNOWLEDGMENTS}

F.K.A. and B.A.M. appreciate the hospitality of Instituto de Fisica Teorica - UNESP (São Paulo, Brazil). The work was partially supported by FAPESP. The authors are grateful to R. Galimzyanov for help with the numerical simulations.

\section{APPENDIX A: CALCULATION OF THE MOMENTS}

For the modulation analysis of Sec. III B, we introduce the following integrals involving the Townes soliton.

The boundary value problem (25) has been solved by discretizing using finite differences and using the shooting method. The solutions give a residual smaller than $10^{-7}$.

The integrals have been calculated using the trapezoidal rule. As a test the following integrals has been calculated, the norm $N\left(R_{s}\right)=1.862 \ldots$ and Hamiltonian $\left[H\left(R_{s}\right)=0\right]$. For the other integrals we obtain

$$
\begin{aligned}
& I_{1}=\int_{0}^{\infty} r^{2} d r R_{T}^{2}=1.7, \quad I_{2}=\int_{0}^{\infty} r d r R_{T}^{2}\left(R_{T}^{\prime}\right)^{2}=2.529, \\
& I_{3}=\int_{0}^{\infty} r d r R_{T}^{4}\left(R_{T}^{\prime}\right)^{2}=5.730 \\
& I_{4}=\int_{0}^{\infty} r^{2} d r R_{T}^{3}\left(R_{T}^{\prime}\right)^{3}=-3.109 \\
& I_{5}=\int_{0}^{\infty} d r R^{4}=11.472, \quad I_{6}=\int_{0}^{\infty} r d r R_{T}^{6}=11.312, \\
& I_{8}=\int_{0}^{\infty} r d r R_{T}^{8}=39.963 \text {, } \\
& I_{9}=\int_{0}^{\infty} r d r R_{T}^{3}\left(R_{T}^{\prime}\right)^{3}=-4.872, \\
& I_{10}=\int_{0}^{\infty} r d r R_{T}^{3}\left(R_{T}^{\prime}\right)^{2}=3.669, \\
& I_{11}=\int_{0}^{\infty} r d r R_{T}^{2}\left(R_{T}^{\prime}\right)^{3}=-2.314
\end{aligned}
$$

\section{APPENDIX B: NUMERICAL PROCEDURE FOR SOLVING THE PARTIAL DIFFERENTIAL EQUATION}

Following Ref. [22], we have solved the cylindrical NLS equation (2) using the method of lines where the solution is advanced in time using an ODE solver and the spatial part is discretized using finite differences. Because of its implicit character, this method allows for great stability and accuracy as well as giving the possibility of implementing directly the cylindrical Laplacian and its associated boundary conditions.

Specifically we use as ODE solver the variable step Runge-Kutta of order 4-5 DOPRI5 [26], which enables to control the error made at each step and bound it by a given tolerance. For all the runs presented, the relative error at each 
step is below $10^{-8}$. The cylindrical Laplacian $\partial_{r}^{2}+(D$ $-1) \partial_{r} / r$ is approximated at each node $n$ of the grid using the following formulas:

$$
\begin{aligned}
\left.\psi_{r}\right|_{n}= & \frac{1}{12 h}\left(\psi_{n-2}-8 \psi_{n-1}+8 \psi_{n+1}-\psi_{n+2}\right)+O\left(h^{4}\right), \\
\left.\psi_{r r}\right|_{n}= & \frac{1}{12 h^{2}}\left(-\psi_{n-2}+16 \psi_{n-1}-30 \psi_{n}+16 \psi_{n+1}-\psi_{n+2}\right) \\
& +O\left(h^{4}\right)
\end{aligned}
$$

where $h$ is the mesh size. We have therefore a method to solve Eq. (2) that is $O\left(d t^{4}, h^{4}\right)$.

The first node corresponds to $r=0$ and to its left we introduce two fictitious points so that $\psi_{r_{-}} r=0=0$. At the right-hand-side, boundary chosen sufficiently far from the pulse, $\psi$ was set to be 0 , again in two points.

The number of mesh points was 4000 and the tolerance of the integrator was set to $10^{-8}$. In all cases the $L^{2}$ norm $N$ was conserved up to $10^{-8}$ in relative error as was the Hamiltonian in the absence of modulation. The latter quantity provided an accurate indicator of collapse.
[1] F. Dalfovo, St. Giorgini, L.P. Pitaevskii, and S. Stringari, Rev. Mod. Phys. 71, 463 (1999).

[2] L. Khaykovich, F. Schrek, F. Ferrari, T. Bourdel, J. Cubizolles, L.D. Carr, Y. Castin, and C. Salomon, Science 296, 1290 (2002); K.E. Strecker, G.B. Partrage, A.G. Truscott, and R.G. Hulet, Nature (London) 417, 150 (2002).

[3] L. Bergé, Phys. Rep. 303, 259 (1998).

[4] S.L. Cornish, N.R. Claussen, J.L. Roberts, E.A. Cornell, and C.E. Wieman, Phys. Rev. Lett. 85, 1795 (2000).

[5] Y. Kagan, E.L. Surkov, and G.V. Shlyapnikov, Phys. Rev. Lett. 79, 2604 (1997).

[6] L. Bergé, V.K. Mezentsev, J. Juul Rasmussen, P.L. Christiansen, and Yu.B. Gaididei, Opt. Lett. 25, 1037 (2000).

[7] I. Towers and B.A. Malomed, J. Opt. Soc. Am. B 19, 537 (2002).

[8] Y. Silberberg, Opt. Lett. 15, 1281 (1990); B.A. Malomed, P. Drummond, H. He, A. Berntson, D. Anderson, and M. Lisak, Phys. Rev. E 56, 4725 (1997).

[9] R. Dum, A. Sampera, K. Suominen, M. Brewczyk, M. Kus, K. Rzazevski, and M. Lewenstein, Phys. Rev. Lett. 80, 3899 (1998).

[10] F.Kh. Abdullaev and R.A. Kraenkel, Phys. Lett. A 272, 395 (2000).

[11] F.Kh. Abdullaev, J. Bronski, and R. Galimzyanov, e-print cond-mat/0205464.
[12] S. Adhikari, e-print cond-mat/0207579.

[13] D.H. Dunlap and V.M. Kenkre, Phys. Rev. B 34, 3625 (1986).

[14] R. Graham, M. Schlautmann, and P. Zoller, Phys. Rev. A 45, R19 (1992).

[15] F.L. Moore, J.C. Robinson, C. Bharucha, P.E. Williams, and M.G. Raizen, Phys. Rev. Lett. 73, 2974 (1994).

[16] R.A. Battye, N.R. Cooper, and P.M. Sutcliffe, Phys. Rev. Lett. 88, 080401 (2002).

[17] D. Anderson, Phys. Rev. A 27, 1393 (1983).

[18] B.A. Malomed, Prog. Opt. 43, 69 (2002).

[19] V.M. Pérez-García, H. Michinel, J.I. Cirac, M. Lewenstein, and P. Zoller, Phys. Rev. A 56, 1424 (1997).

[20] Yu. Kivshar and S. Turitsyn, Phys. Rev. E 49, 2536 (1994).

[21] T.S. Yang and W.L. Kath, Opt. Lett. 22, 985 (1997).

[22] G. Fibich and G.C. Papanicolaou, Phys. Lett. A 239, 167 (1998); SIAM (Soc. Ind. Appl. Math.) J. Appl. Math. 60, 183 (1999).

[23] J.H.B. Nijhof, N.J. Doran, W. Forysiak, and F.M. Knox, Electron. Lett. 33, 1726 (1997).

[24] F.Kh. Abdullaev and J.G. Caputo, Phys. Rev. E 58, 6637 (1998).

[25] L. Bergé and A. Couairon, Physica D 152-153, 752 (2001).

[26] E. Hairer, S.P. Norsett, and G. Wanner, Solving Ordinary Differential Equations I (Springer-Verlag, Berlin, 1987).

[27] I. Towers and B.A. Malomed (unpublished). 